\title{
An interpretation of light curves of SV Camelopardalis by the inverse-problem method
}

\author{
G. Djurašević \\ Astronomical Observatory, Belgrade, Yugoslavia \\ e-mail: gdjurasevic@aob.aob.bg.ac.yu
}

Received January 27; accepted May 12, 1997

\begin{abstract}
The paper is devoted to the problem of the determination of the orbital and physical parameters of the active eclipsing binary $S V$ Cam on the basis of the interpretation of photometric observations made by Patkós (1982) during the period 1973-1981. The problem is solved in two stages: by obtaining a synthetic light curve in the case when the parameters of the corresponding Roche model (Djurašević 1992a) are given a priori (direct problem), and by determining the parameters of the model for which the best fit between the synthetic light curve and the observations is achieved (inverse problem) (Djurašević 1992b). A total of 18 light curves are analysed in the framework of the Roche model, involving one and two spotted regions on the primary component of the system (Sp G3 V), for the temperature contrast between the spotted area and the surrounding photosphere $A_{\mathrm{s}}=T_{\mathrm{s}} / T_{1}=$ 0.65 . The basic parameters of the system and of the spotted areas are estimated. Throughout the whole set of the analysed light curves, a double spot model fits the observations satisfactorily. A single spot model yields a poorer fit, where the basic system parameters obtained by analysing the individual light curves show stronger variations about a mean value. That indicates that the single spot model cannot successfully reproduce the $S V$ Cam light curve changes during the analysed period.
\end{abstract}

According to the obtained results the spotted areas are formed at high latitudes and cover a significant part of the stellar surface. No clear cyclicity of the system's activity is noted from the analysed observations. There are some indications that spotted areas at high latitudes (above $70^{\circ}$ ) correspond to an enhanced activity. Since the system's period is short $(P=0.59)$, the presence of spotted regions at high latitudes can be explained by the dynamo mechanism for rapid rotators (Schüssler \& Solanski 1992). During the analysed period the spotted areas tend to fall into a specially active longitude sectors at high latitudes, near stellar polar regions. Due to a selection effect

Send offprint requests to: G. Djurašević it is possible that a more extensive observational material would correct this result to some extent.

The light curve analysis allowed an estimation of the system parameters and of the active spotted regions.

Key words: stars: activity; eclipsing binaries-stars; individual: $S V$ Cam - methods data analysis

\section{Introduction}

The Close Binary (CB) SV Cam has an orbital period $P=0$ d59307. According to Hall (1976), it belongs to the short-period group of the $R S C V n$ binaries. Hilditch et al. (1979) found that this system is composed of a G3V primary, and $\mathrm{K} 4 \mathrm{~V}$ secondary, with the mass ratio $q=$ $m_{2} / m_{1}=0.7$. They made the hypothesis that the light variations are due to a $B Y$ Draconis-type variability of the K4V secondary. The careful photometry by Patkós (1982) demonstrated conclusively the existence of a "distortion wave", which migrates toward increasing orbital phase, and strong flaring activity, which Patkós (1981) attributed to the active regions on the secondary star.

Using the Patkós' (1982) observations, Busso et al. (1985) analysed the differential rotation problem on the basis of the depression migration (caused by spotted areas) through the light curve orbital phase with time. By using a simplified model, the authors estimated the relevant parameters, such as the angular velocity at the equator, the differential rotation rate, the mean velocity of the meridional motion, the latitude of spots at formation and the corotation latitude.

Observations extending over about half a century (1932-1984) were analysed by Zeilik et al. (1988) in the framework of the spot modelling technique (Budding 1977; Budding \& Zeilik 1987) with spots on the primary. According to these authors, the active dark spotted region covers a significant part of the stellar surface occupying high latitudes (about $60^{\circ}-75^{\circ}$ ). The single active 
region tends to fall into two longitude sectors, $45^{\circ}-135^{\circ}$ and $225^{\circ}-315^{\circ}$. These resemble the active longitude belts such as proposed by Eaton \& Hall (1979) for the $R S C V n$ group in general.

\section{The spot model and the inverse problem method}

For the purpose of analysing the asymmetric light curves deformed by the presence of spotted areas on the components a Roche model based on the principles originated in the paper by Wilson \& Devinney (1971) is developed. Here only some basic elements of the model are indicated, whereas the details can be found in Djurašević (1992a).

The stellar size in the model is described by the filling coefficients for the critical Roche lobes $F_{1,2}$ of the primary and secondary respectively. They indicate to what degree the stars of the system fill the corresponding critical lobes (see Fig. 1). In the case of synchronous rotation of the components these coefficients are expressed through the ratio of the stellar polar radii, $R_{1,2}$, and the corresponding polar radii of the critical Roche lobes, i.e. $F_{1,2}=R_{1,2} / R_{\text {Roche }_{1,2}}$. The stellar nonsynchronous rotation is described by coefficients $f_{1,2}=\omega_{1,2} / \omega_{\mathrm{K}}$, where $\omega_{1,2}$ is the angular rotation rate of the components and $\omega_{\mathrm{K}}$ is the Keplerian orbital revolution rate. In this case, the critical Roche lobes belong to the critical nonsynchronous lobes, and the filling coefficients $F_{1,2}$ are defined with respect to their polar radii. For a given mass ratio of the components $q=m_{2} / m_{1}$, and the nonsynchronous rotation coefficients $f_{1,2}$, the stellar shape and size in a CB Roche model are unequivocally determined by the filling coefficients $F_{1,2}$ of the critical lobes (Djurašević 1992a).

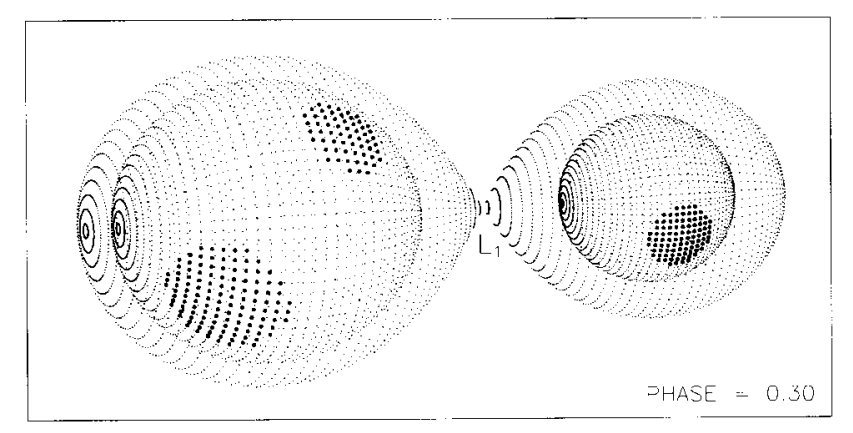

Fig. 1. The Roche model of an active CB with spotted areas on the components

The presence of spotted areas (dark or bright) enables one to explain the asymmetry and the depressions on the light curves of active CBs. In the model these regions are approximated by circular spots (Fig. 1) characterised by the temperature contrast of the spot with respect to the surrounding photosphere $\left(A_{\mathrm{s}}=T_{\mathrm{s}} / T_{*}\right)$, by the angular dimensions (radius) of the spot $(\theta)$ and by the longitude $(\lambda)$ and latitude $(\varphi)$ of the spot centre.

For a successful application of the model in analysing the observed light curves an efficient method unifying the best properties of the Steepest Descent and the Differential Corrections method into a single algorithm (Djurašević 1992b) is proposed. This method is obtained by modifying the Marquardt's (1963) algorithm.

The interpretation of photometric observations is based on the choice of optimal model parameters yielding the best agreement between the observed light curve and the corresponding synthetic one. Some of these parameters can be determined a priori in an independent way, while the others are found by solving the inverse problem. Typical case of the inverse problem involves the estimate of the following parameters: mass ratio of the components $\left(q=m_{2} / m_{1}\right)$, filling coefficients of the critical Roche lobes $\left(F_{1,2}\right)$, orbit inclination $(i)$, temperature of either component $(T)$ and spotted areas parameters $(\theta, \lambda$ and $\varphi)$. The temperature contrast of the spotted regions with respect to the surrounding photosphere $\left(A_{\mathrm{s}}\right)$ is usually given a priori.

The Roche model given above and the method for the inverse-problem solution allow us to obtain a direct analysis of the observed light curves.

\section{Procedure of light curve analysis}

The present paper contains an analysis of the light curves of $S V$ Cam observed in the period 1973-1981 (Patkós 1982), which is based on the Roche model with spotted areas on the components (Djurašević 1992a). In Table 1 the data sequence of the individual light curves is presented. Here only the $V$ passband light curves are considered because they are the most complete. Out of a total of 38 light curves, 18 (which are relatively well defined) are chosen to enable the estimate of the system and spotted areas parameters. There are indications that they all belong to one activity cycle (Busso et al. 1985).

In the analysis of the light curves, we avoided the somewhat questionable practice of forming normal points, and included all observations. We obtained the optimal model parameters trough the minimization of $S=\Sigma(\mathrm{O}-\mathrm{C})^{2}$, where $\mathrm{O}-\mathrm{C}$ is the residual between observed (LCO) and synthetic (LCC) light curves for a given orbital phase. The minimization of $S$ is done in an iterative cycle of corrections of the model parameters, based on the Marquardt's algorithm. This inverse problem method is characterised by fast and reliable convergence, that allows one to efficiently estimate the system parameters. The method also gives standard errors.

Since the results of the light curve analysis are very dependent on the choice of the adopted working hypothesis, the analysis is carried out within the framework of several hypotheses (single and double spotted areas; spots on the primary and spots on the secondary). 
The present light curve analysis yields the filling coefficients of the critical Roche lobes $F_{1}$ and $F_{2}$, about 0.88 and 0.64 for the primary and secondary, respectively (see Tables 2 and 3). Since the critical Roche lobes are filled by the components to a high degree, tidal effects are expected to contribute to synchronisation of the rotational and orbital periods. Therefore, in solving the inverse problem, for nonsynchronous rotation coefficients we adopted the values $f_{1,2}=1.0$.

For such a case where neither the primary nor the secondary fill the critical Roche lobes, determination of the mass ratio of the components by analysing light curves only is not reliable. For this reason the mass ratio is fixed by assuming the value $q=m_{2} / m_{1}=0.71$, estimated spectroscopically (Budding \& Zeilik 1987). On the basis of its spectral type $(\mathrm{G} 3 \mathrm{~V})$ the temperature of the primary is also fixed $\left(T_{1}=5800 \mathrm{~K}\right)$.

In the programme for solving the inverse problem, the linear limb-darkening coefficients are determined on the basis of the stellar effective temperature and of the stellarsurface gravity, according to the given spectral type, by using the polynomial proposed by Díaz-Cordovés et al. (1995). For the gravity-darkening coefficients of the stars the value of $\beta_{1,2}=0.08$ was adopted. Lucy (1967) and Osaki (1970) regard this value as being justifiable for stars with convective envelopes.

The temperature of the secondary (about $4300 \mathrm{~K}$ ) was significantly lower than that of the primary. Therefore, its contribution to the total brightness of the system is relatively small. Hence one can expect that the spotted areas on the secondary yield comparatively small photometric effects. In this case, the model for the light curve fitting requires very large spotted regions with a high temperature contrast with respect to the surrounding photosphere. The spotted areas are too large even for a temperature contrast $A_{\mathrm{s}}=T_{\mathrm{s}} / T_{2}=0.6$, which yields the spot temperature of about $2600 \mathrm{~K}$. In the case of some light curves analysed here the fitting of observations with a synthetic light curve is not satisfactory. The basic parameters of the system, such as the size of the components, the orbit inclination and the temperature of the secondary obtained by analysing the individual light curves, should be variable within significant limits, which is unacceptable. Therefore, this hypothesis is rejected as unrealistic.

Under the assumption of spotted areas being on the primary, the optimum synthetic light curves fit much better the observations. The light curves were then analysed in the framework of the single and double spot models. For the temperature contrast between the spotted area and the surrounding photosphere it is assumed $A_{\mathrm{s}}=T_{\mathrm{s}} / T_{1}=0.65$, yielding the spot temperature to be about $3770 \mathrm{~K}$. Cellino et al. (1985) inferred from the infrared observations a spot temperature of $3780 \mathrm{~K}$. On the basis of this result the assumed value for the spotted area temperature contrast may be considered as justified.
In analysing the light curves the following procedure is applied. First, on the basis of the light curve form, the curve No. 10 (see Tables 1-3 and Fig. 3) was chosen as the cleanest from spot effects. In analysing it, the optimisation begins using only the basic model parameters. After achieving a first convergence, one also includes free parameters related to spots in the iterative optimisation process.

The basic parameters of the system, obtained in this way, are used as starting points in the inverse-problem solution for other light curves. Their analysis begun by optimisation in the spot parameters. When the optimisation based on these parameters does not secure a further minimization of $\Sigma(\mathrm{O}-\mathrm{C})^{2}$, the basic system parameters have to be introduced in the iterative process. Namely, one cannot in advance exclude the possibility of certain changes of some of these parameters during the analysed period of time. Using this procedure we optimize all freeparameters of the model in the final iterations. In this way we save some computer time because a smaller number of iterations is needed.

\section{Results}

The results of the light curve analysis in a "compressed" form are presented in Table 2 (single spot model) and Table 3 (double spot model). The tables contain parameters of the system evaluated through an analysis of individual light curves, numerated in accordance with Table 1. The errors in parameter estimation originated from the nonlinear least-squares method on which the inverse problem method is based. They are just formal and do not represent a real accuracy of the evaluation of the parameters. Especially, in evaluation of the spotted area size and latitude the real errors are probably larger than mentioned. This may be explained by the lack of a method for estimating the spot latitude and dimensions on the basis of the light curve analysis. Namely, for a given orbit inclination, light curve modulations produced by a smaller spotted area at a lower latitude or by a larger spotted area at a higher latitude are similar. This gives rise to errors in the spot-latitude and spot size estimation. In well-defined light curves the real spot size and latitude errors approximately estimated amount to $\Delta \theta= \pm 2^{\circ}$ and $\Delta \varphi= \pm 10^{\circ}$. The errors can be larger in light curves insufficiently covered by observations.

On the basis of the results presented in Tables 2 and 3 , and from Fig. 2a, it is evident that the double spot model yields a better fit (lesser $\left.\Sigma(\mathrm{O}-\mathrm{C})^{2}\right)$. In this case, the basic system parameters are approximately constant for the whole set of the analysed light curves (see Figs. $2 \mathrm{~b}-\mathrm{d}$ ). This means that for the entire observational period the changes in the light curve form can be almost completely explained by changes in the position and size of the spotted areas. Certain variability can be noticed in temperature of the secondary, with the minimum value 

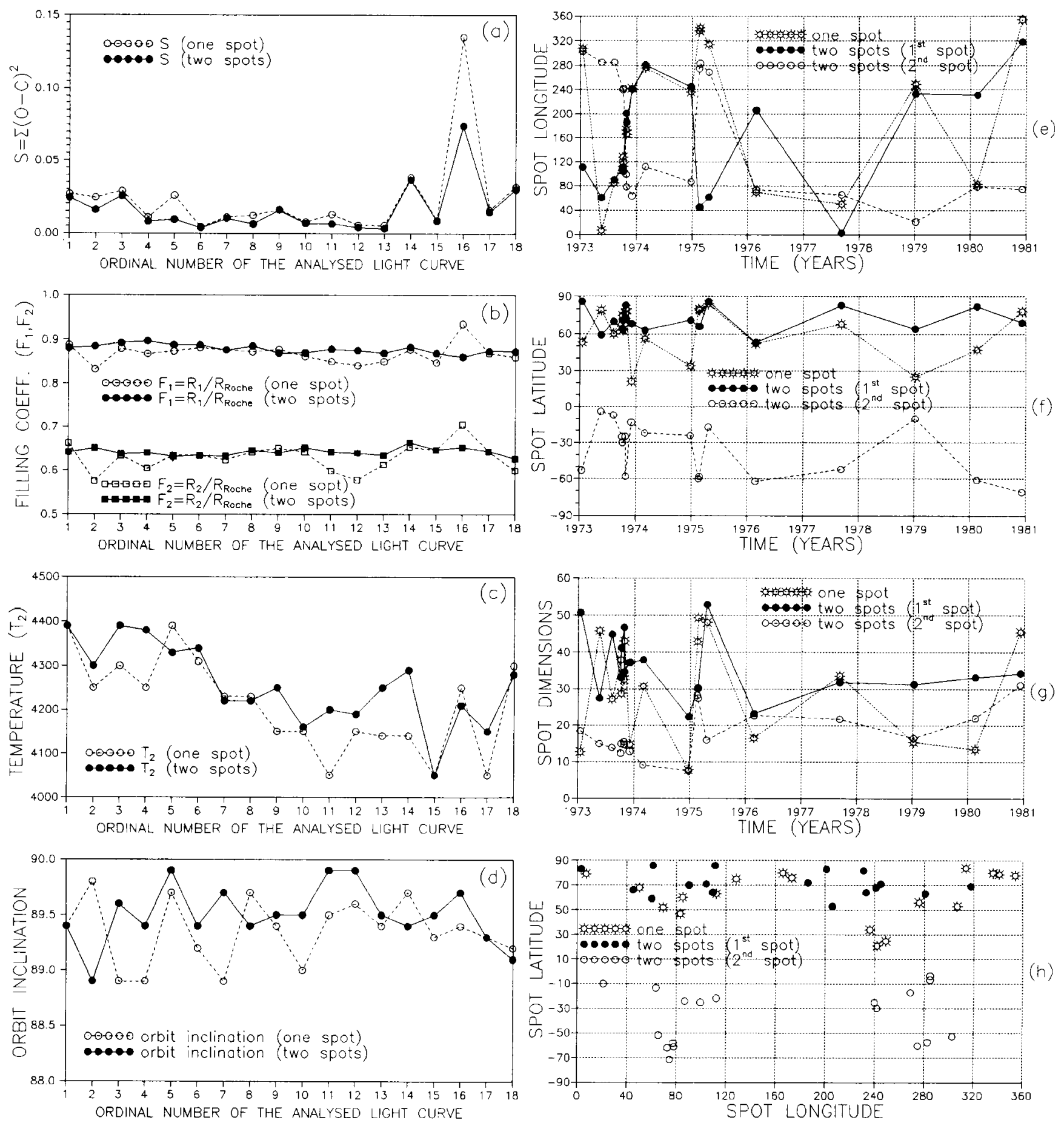

Fig. 2. Solutions obtained by analysing the light curves of $S V$ Cam within the framework of the Roche model with one and two spotted areas: Left - Quality of fitting $(\mathrm{S})$ and basic sistem parametrs $\left(F_{1,2}, T_{2}, i\right)$ obtained by analysing the individual light curves; Right - spotted area parametres $(\lambda, \varphi$ and $\theta)$ and $(\lambda, \varphi)$ spot positions during the period 1973-1981 
in light curve No. 15. A comparatively small depth of the secondary minimum of this light curve, probably indicates a real phenomenon. The variations of the orbit inclination are within the accuracy limits of the evaluation of this parameter.

In the case of the single spot model, the fit obtained is somewhat poorer, whereas the basic system parameters obtained by analysing the individual light curves show stronger variations about a mean value (see Table 2 and Fig. 2 - left column). We consider this case as less reliable, to the point of being possibly excluded.

The change of active-region parameters over the analysed observational period is suitable to be presented on plots. Such a presentation is given in Fig. 2 (right column), for a single and double spot model. The spot migration in longitude during the analysed period is shown in Fig. 2e. In the double spot model differences in spot longitudes in opposite stellar hemispheres can be noticed.

The spotted areas appear at high latitudes, near the polar regions (Fig. 2f). In the single spot model, for all light curves one obtains a spotted area on the upper stellar hemisphere, at sufficiently high latitudes $\left(20^{\circ}-80^{\circ}\right)$. In the case of the double spot model, the $1^{\text {st }}$ larger spotted area is on the upper stellar hemisphere, near the polar region (latitudes $50^{\circ}-86^{\circ}$ ). The $2^{\text {nd }}$ smaller spotted area is on the lower sttelar hemisphere, with latitudes in the interval $\left(-5^{\circ}--70^{\circ}\right)$. During the analysed period, the angular distance beetwen the centres of these two spotted areas is in the interval $62^{\circ}$ to $178^{\circ}$ in longitude and $63^{\circ}$ to $143^{\circ}$ in latitude. The mean values of these distances amount to $\Delta \lambda_{1,2}=137^{\circ}$ and $\Delta \varphi_{1,2}=107^{\circ}$.

The size of spotted area can be an indicator of the system's activity. Based on the obtained results (Fig. 2g) one can say that the system during 1973 showed a significant activity. During 1974 the activity decreased. Therefore, for light curve No. 10, obtained in late 1974, one finds minimum dimensions of the spotted areas. After this, there is a fast increase in the activity. In 1976 it reaches a lower level again, at which it remains with smaller changes till the end of 1980. It seems that then a new significant increase in the activity took place. Then the activity increases again. Unfortunately, the data available are not sufficiently dense in time to study the activity in more detail. A clear cyclicity in the system's activity is not noticeable.

In the framework of the obtained solutions for both models it is possible to see a correlation between the latitude, (Fig. 2f) and size (Fig. 2g) of spotted areas. The large spotted area, near the stellar polar regions corresponds to an enhanced activity of the system.

During the analysed period, $(\lambda, \varphi)$-postions of the spotted area are grouped within active longitude and latitude sectors (Fig. 2h). For the single spot model they are in the intervals $\left(50^{\circ}-170^{\circ}\right)$ and $\left(230^{\circ}-365^{\circ}\right)$ of longitude, and in $\left(20^{\circ}-80^{\circ}\right)$ of latitude respectively. In the case of the double spot model the active longitude belts for $1^{\text {st }}$ spot in the intervals $\left(45^{\circ}-110^{\circ}\right)$ and $\left(200^{\circ}-250^{\circ}\right)$ are less prominent, but more prominent for $2^{\text {nd }}$ spot in the $\left(60^{\circ}-120^{\circ}\right)$ and $\left(240^{\circ}-300^{\circ}\right)$ ones. The latitudes are concentrated within the sectors $\left(50^{\circ}-85^{\circ}\right)-\left(1^{\text {st }}\right.$ spot $)$ and $\left(-5^{\circ}--70^{\circ}\right)-\left(2^{\text {nd }}\right.$ spot $)$. Due to a selection effect it is possible that a more extensive observational material would correct this result to some extent.

Photometric effects of the spots with longitudes about $0^{\circ}$ and $180^{\circ}$ would be observable from the depth and shape of the light curve minima and partialy from the rest part of the light curve, which is not covered by eclipses. So, masking by eclipses does not explain the noticeable scarcity of these spots.

The obtained fit of the observed light curves (LCO) by the synthetic ones (LCC) following from the inverse problem solutions based on the single and double spot model are shown in Fig. 3. In order to easily follow the obtained solutions, the light curves are noted by ordinal numbers (No) in accordance with the ones applied in Tables 1, 2 and 3. Substantial differences in the quality of fits obtained by using single (dashed line) and double spot models (solid line) can be noticed in some light curves.

Figure 4 (double spot model) shows the view of the system obtained on the basis of the parameters estimated by analysing the corresponding light curves. The numeration of the figures corresponds to the ordinal number of the analysed light curves. The figures were made by using the programme (Djurašević 1991). Thanks to such plots, one can see a view of a CB system at a noted orbital phase, chosen in such a way that the spots are visible.

\section{Discussion and conclusions}

Both presentations of the results (Table 3 and corresponding figures) show that in the case of the Roche model with two spotted regions, the synthetic light curves obtained by solving the inverse problem fit the observations very well (almost within the measurement accuracy). The variations of the basic system parameters among the different curves for the analysed period are insignificant. This means that the variations in the light curves can be explained by the change of the position and size of the spotted areas on the primary.

For the majority of the analysed light curves the single spot model (Table 2 and corresponding figures) fits well the observations. But this model requires comparatively large variations of the basic system parameters (e.g. the stellar size - Fig. 2b) during the analysed period. Therefore, we tend to exclude this solution.

Appearing at high latitudes, the spotted areas cover a significant part of the stellar surface. Since the system's period is short $(P=0.59)$, the presence of spots at high latitudes (near the pollar regions) can be explained by the dynamo mechanism for rapid rotators (Schüssler \& Solanski 1992).

In favour of presence of spotted areas at high latitudes we have an independent argument based on the 

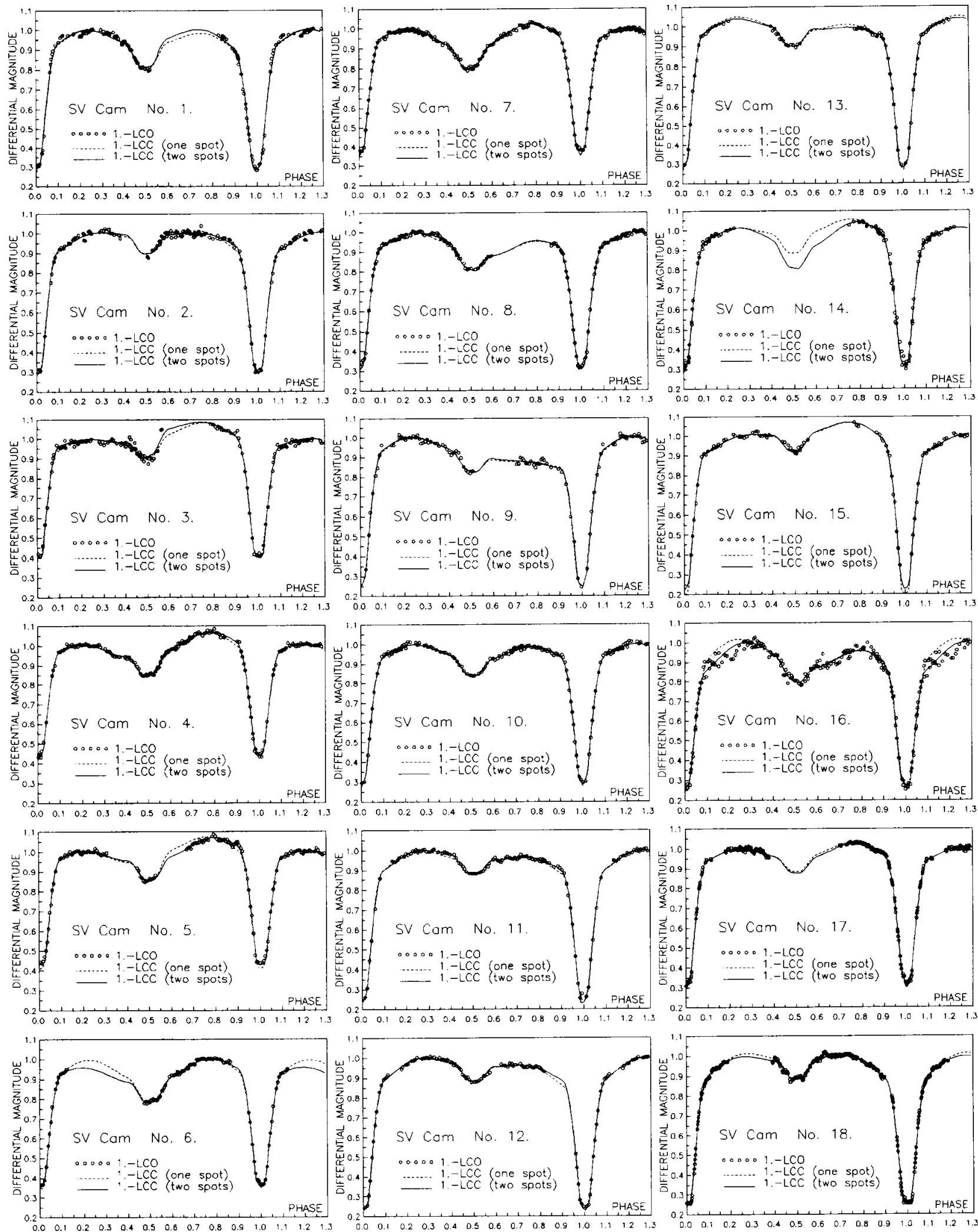

Fig. 3. Observed (LCO) and final synthetic (LCC) light curves of $S V$ Cam obtained by solving the inverse problem within the framework of the Roche model with one (dashed line) and two (solid line) spotted areas 
Table 1. Data sources for the analysis of the observed light curves (Patkós 1982) for the active CB $S V$ Cam (V-filter)

\begin{tabular}{|c|c|c|c|}
\hline Data set No. & Date & JD \\
\hline 1 & 1973 Jan. 04 - 1973 Jan. 12 & 98 \\
2 & 1973 May. 04 - 1973 Jun. 02 & $2441807,2441810,2441824,2441825,2441831,2441833,2441835$ \\
3 & 1973 Aug. 05 - 1973 Aug. 11 & $2441900,2441901,2441903,2441904,2441905$ \\
4 & 1973 Oct. 03 - 1973 Oct. 06 & $2441959,2441960,2441961$ \\
5 & 1973 Oct. 06 - 1973 Oct. 08 & 2441962,2441963 \\
6 & 1973 Oct. 26 - 1973 Oct. 27 & 2441982 \\
7 & 1973 Oct. 27 - 1973 Oct. 29 & 2441983,2441984 \\
8 & 1973 Dec. 02 - 1973 Dec. 03 & 2442019 \\
9 & 1974 Feb. 27 - 1974 Mar. 02 & 2442106,2442108 \\
10 & 1974 Dec. 22 - 1974 Dec. 24 & 2442404,2442405 \\
11 & 1975 Feb. 16 - 1975 Feb. 18 & $2442432,2442460,2442461$ \\
12 & 1975 Feb. 21 - 1975 Feb. 23 & 2442465,2442466 \\
13 & 1975 Apr. 19 - 1975 Apr. 21 & 2442522,2442523 \\
14 & 1976 Feb. 20 - 1976 Feb. 28 & $2442830,2442831,2442836$ \\
15 & 1977 Sep. 05 - 1977 Sep. 08 & $2443392,2443393,2443394$ \\
16 & 1979 Jan. 04 - 1979 Jan. 07 & $2443878,2443879,2443880$ \\
17 & 1980 Feb. 15 - 1980 Feb. 16 & 2444285 \\
18 & 1980 Dec. 08 - 1980 Dec. 09 & 2444582 \\
\hline
\end{tabular}

Note: JD - Julian Dates of the observations, $N$ - total number of observations for the given light curve.

Table 2. Results of the analysis of the $S V$ Cam light curves obtained by solving the inverse problem for the Roche model with one spotted area on the primary component

\begin{tabular}{|c|c|c|c|c|c|c|c|c|c|c|c|c|c|}
\hline No. & $\theta$ & $\lambda$ & $\varphi$ & $F_{1}$ & $F_{2}$ & $T_{2}$ & $i$ & $u_{2}$ & $\Omega_{1}$ & $\Omega_{2}$ & $R_{1}$ & $R_{2}$ & $\Sigma(\mathrm{O}-\mathrm{C})^{2}$ \\
\hline 1 & $12.7 \pm 1.3$ & $307 \pm 11$ & $53 \pm 7$ & $0.888 \pm 0.005$ & $0.662 \pm 0.004$ & $4390 \pm 50$ & $89.4 \pm 1.4$ & 0.76 & 3.559 & 4.394 & 0.342 & 0.217 & 0.02663 \\
2 & $45.7 \pm 1.0$ & $7 \pm 2$ & $79 \pm 1$ & $0.833 \pm 0.005$ & $0.575 \pm 0.003$ & $4250 \pm 70$ & $89.8 \pm 0.8$ & 0.77 & 3.796 & 4.897 & 0.321 & 0.188 & 0.02422 \\
3 & $27.2 \pm 0.7$ & $85 \pm 3$ & $60 \pm 1$ & $0.880 \pm 0.006$ & $0.634 \pm 0.005$ & $4300 \pm 60$ & $88.9 \pm 0.9$ & 0.76 & 3.626 & 4.540 & 0.339 & 0.208 & 0.02871 \\
4 & $37.9 \pm 0.5$ & $128 \pm 1$ & $75 \pm 1$ & $0.868 \pm 0.003$ & $0.604 \pm 0.002$ & $4250 \pm 30$ & $88.9 \pm 0.5$ & 0.77 & 3.667 & 4.710 & 0.334 & 0.198 & 0.01090 \\
5 & $28.7 \pm 0.6$ & $112 \pm 4$ & $63 \pm 1$ & $0.874 \pm 0.007$ & $0.634 \pm 0.004$ & $4390 \pm 50$ & $89.7 \pm 0.9$ & 0.76 & 3.645 & 4.540 & 0.336 & 0.208 & 0.02555 \\
6 & $32.5 \pm 0.9$ & $173 \pm 4$ & $76 \pm 1$ & $0.882 \pm 0.003$ & $0.634 \pm 0.002$ & $4310 \pm 30$ & $89.2 \pm 0.9$ & 0.76 & 3.619 & 4.542 & 0.339 & 0.208 & 0.00447 \\
7 & $43.0 \pm 1.0$ & $166 \pm 2$ & $80 \pm 1$ & $0.877 \pm 0.003$ & $0.624 \pm 0.002$ & $4230 \pm 30$ & $88.9 \pm 0.6$ & 0.77 & 3.634 & 4.597 & 0.338 & 0.205 & 0.01095 \\
8 & $14.7 \pm 0.8$ & $242 \pm 3$ & $21 \pm 14$ & $0.873 \pm 0.003$ & $0.642 \pm 0.002$ & $4230 \pm 40$ & $89.7 \pm 1.2$ & 0.77 & 3.648 & 4.496 & 0.336 & 0.211 & 0.01224 \\
9 & $30.7 \pm 0.6$ & $276 \pm 3$ & $56 \pm 11$ & $0.878 \pm 0.009$ & $0.651 \pm 0.006$ & $4150 \pm 80$ & $89.4 \pm 1.0$ & 0.78 & 3.633 & 4.452 & 0.338 & 0.213 & 0.01615 \\
10 & $7.8 \pm 0.6$ & $236 \pm 9$ & $34 \pm 10$ & $0.863 \pm 0.003$ & $0.642 \pm 0.002$ & $4150 \pm 40$ & $89.0 \pm 1.3$ & 0.78 & 3.686 & 4.497 & 0.332 & 0.210 & 0.00775 \\
11 & $42.9 \pm 0.8$ & $336 \pm 2$ & $80 \pm 1$ & $0.851 \pm 0.004$ & $0.599 \pm 0.003$ & $4050 \pm 60$ & $89.5 \pm 0.9$ & 0.79 & 3.728 & 4.742 & 0.327 & 0.196 & 0.01292 \\
12 & $49.3 \pm 0.5$ & $341 \pm 2$ & $79 \pm 1$ & $0.842 \pm 0.003$ & $0.578 \pm 0.002$ & $4150 \pm 30$ & $89.6 \pm 0.3$ & 0.78 & 3.762 & 4.877 & 0.324 & 0.189 & 0.00554 \\
13 & $48.1 \pm 0.8$ & $314 \pm 7$ & $84 \pm 1$ & $0.851 \pm 0.005$ & $0.613 \pm 0.003$ & $4140 \pm 50$ & $89.4 \pm 1.1$ & 0.78 & 3.726 & 4.656 & 0.328 & 0.201 & 0.00530 \\
14 & $16.5 \pm 1.2$ & $69 \pm 7$ & $52 \pm 4$ & $0.878 \pm 0.006$ & $0.654 \pm 0.004$ & $4140 \pm 110$ & $89.7 \pm 2.3$ & 0.78 & 3.631 & 4.436 & 0.338 & 0.214 & 0.03825 \\
15 & $33.6 \pm 0.9$ & $50 \pm 2$ & $68 \pm 1$ & $0.849 \pm 0.008$ & $0.647 \pm 0.005$ & $4050 \pm 60$ & $89.3 \pm 1.2$ & 0.79 & 3.736 & 4.472 & 0.327 & 0.212 & 0.00884 \\
16 & $15.4 \pm 0.8$ & $249 \pm 6$ & $25 \pm 8$ & $0.936 \pm 0.008$ & $0.706 \pm 0.006$ & $4250 \pm 80$ & $89.4 \pm 1.4$ & 0.77 & 3.445 & 4.187 & 0.360 & 0.231 & 0.13520 \\
17 & $13.4 \pm 0.3$ & $83 \pm 3$ & $47 \pm 2$ & $0.870 \pm 0.002$ & $0.644 \pm 0.002$ & $4050 \pm 50$ & $89.3 \pm 0.5$ & 0.79 & 3.660 & 4.487 & 0.335 & 0.211 & 0.01586 \\
18 & $45.5 \pm 0.4$ & $354 \pm 1$ & $78 \pm 1$ & $0.861 \pm 0.002$ & $0.600 \pm 0.002$ & $4300 \pm 20$ & $89.2 \pm 0.3$ & 0.76 & 3.693 & 4.739 & 0.331 & 0.197 & 0.03177 \\
\hline
\end{tabular}

Fixed parameters:

$q=m_{2} / m_{1}=0.71$ - mass ratio of the components,

$T_{1}=5800 \mathrm{~K}$ - temperature of the primary,

$A_{\mathrm{s}}=T_{\mathrm{s}} / T_{1}=0.65$ - spotted area temperature coefficient,

$f_{1}=f_{2}=1.00$ - nonsynchronous rotation coefficients of the components,

$\beta_{1}=\beta_{2}=0.08$ - gravity-darkening coefficients of the components,

$u_{1}=0.66$ - limb-darkening coefficient of the primary.

Note: No. - data set No., $\theta$ - spotted area angular dimensions, $\lambda$ - spot longitude and $\varphi$ - spot latitude (all in degrees), $F_{1}, F_{2}$ - filling coeficients for critical Roche lobes of the primary and secondary, $T_{2}$ - temperature of the secondary, $i-$ orbit inclination (in degrees), $u_{2}-$ limb-darkening coefficient of the secondary, $\Omega_{1}, \Omega_{2}$ - dimensionless surface potentials of the primary and secondary, $R_{1}, R_{2}-$ stellar polar radii in units of the distance between the component centres and $\Sigma(\mathrm{O}-\mathrm{C})^{2}$ - final sum of squares of residuals between observed (LCO) and synthetic (LCC) light curves. 
Table 3. Results of the analysis of the $S V$ Cam light curves obtained by solving the inverse problem for the Roche model with two spotted areas on the primary component

\begin{tabular}{|c|c|c|c|c|c|c|c|c|c|c|c|c|c|c|c|c|}
\hline No. & $\theta_{1}$ & $\lambda_{1}$ & & $\theta_{2}$ & $\lambda_{2}$ & & $F_{1}$ & $F_{2}$ & $T_{2}$ & $i$ & $a_{2}$ & $\Omega_{1}$ & $\Omega_{2}$ & $R_{1}$ & $R_{2}$ & $\Sigma(\mathrm{O}-\mathrm{C})^{2}$ \\
\hline 1 & $50.7 \pm 2.2$ & $111 \pm 7$ & $86 \pm 12$ & $3.4 \pm 0.9$ & $303 \pm 5$ & $-53 \pm 3$ & $0.881 \pm 0.006$ & $0.641 \pm 0.004$ & $4390 \pm 50$ & $89.4 \pm 0$. & $\mid 0.76$ & .623 & .404 & 0.339 & 0.210 & 0.02400 \\
\hline 2 & $27.4 \pm 0.4$ & $60 \pm 2$ & $59 \pm 1$ & $\mid 14.9 \pm 0.4$ & $285 \pm 2$ & $-4 \pm 3$ & $85 \pm 0.005$ & $0.651 \pm 0.003$ & $4300 \pm 50$ & $.0 \pm 0.6$ & 0.76 & 607 & 4.450 & .341 & .213 & 0.01575 \\
\hline 3 & $44.8 \pm 0.6$ & $90 \pm 2$ & $70 \pm 1$ & $13.8 \pm 0.7$ & $285 \pm 5$ & $-7 \pm 20$ & $893 \pm 0.006$ & $0.638 \pm 0.005$ & $4390 \pm 50$ & $.6 \pm 0.9$ & 0.76 & .583 & 4.516 & 0.344 & 209 & 2531 \\
\hline 4 & $33.1 \pm 0.2$ & $109 \pm 1$ & $64 \pm 1$ & & $240 \pm 3$ & $-25 \pm 3$ & $897 \pm 0.002$ & .002 & & & & 567 & 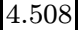 & & 10 & 801 \\
\hline 5 & & $104 \pm 2$ & & & $2 \pm 3$ & $-30 \pm 5$ & & & & & & 3.599 & & & 07 & 928 \\
\hline 6 & & $201 \pm 4$ & & & \pm 3 & & & & & & & & & 42 & 18 & 371 \\
\hline 7 & .6 & $186 \pm 2$ & & & \pm 4 & & & & & & & & & & & 08 \\
\hline 8 & .5 & $241 \pm 2$ & \pm 1 & & \pm 2 & $-13 \pm 4$ & 3 & 02 & 42 & t & 0.77 & 00 & & 41 & 12 & 26 \\
\hline 9 & 0.6 & $281 \pm 3$ & \pm 1 & \pm 1.0 & $112 \pm 6$ & $-22 \pm 25$ & & 05 & & & & 60 & & 35 & 10 & 80 \\
\hline 11 & $30.2 \pm 0.4$ & $45 \pm 1$ & $66 \pm 1$ & \pm 0.3 & $275 \pm 2$ & $-60 \pm 1$ & 03 & 002 & & & 7 & $2 \zeta$ & 4 & 38 & 11 & 351 \\
\hline 12 & $30.2 \pm 0.4$ & $45 \pm 1$ & \pm 1 & \pm 0.3 & $283 \pm 2$ & $-58 \pm 1$ & 03 & 02 & 30 & & 7 & 3.639 & 4 & 37 & 10 & 100 \\
\hline 13 & 52.9 & $61 \pm 4$ & & & $269 \pm 2$ & $-17 \pm 3$ & & & & & & 58 & & 35 & 08 & 332 \\
\hline 14 & & $206 \pm 15$ & \pm 35 & $22.8 \pm 1.3$ & $73 \pm 8$ & $-62 \pm 3$ & & & & & & & & & 18 & 880 \\
\hline 15 & 2.1 & $3 \pm 21$ & & & $65 \pm 2$ & $-52 \pm 3$ & & & & & & & & & & 43 \\
\hline 16 & & $233 \pm 4$ & & $16.5 \pm 0.5$ & \pm 2 & $-10 \pm 6$ & & & & & & & & & & 26 \\
\hline 17 & $33.2 \pm$ & $231 \pm 3$ & & $22.0=$ & \pm 2 & $-61 \pm 1$ & & & & & & 3.642 & & 37 & & 44 \\
\hline 18 & $34.3 \pm 0.4$ & $318 \pm 1$ & \pm 1 & $31.1 \pm 0.4$ & $74 \pm 2$ & & $4 \pm 0.002$ & $7 \pm 0.001$ & 42 & $2 \pm 0.3$ & 0 & 3.64 & 4.58 & 0.337 & 0.205 & 0.02985 \\
\hline
\end{tabular}

Fixed parameters:

$q=m_{2} / m_{1}=0.71-$ mass ratio of the components,

$T_{1}=5800 \mathrm{~K}$ - temperature of the primary,

$A_{\mathrm{s}_{1,2}}=T_{\mathrm{s}_{1,2}} / T_{1}=0.65$ - spotted areas temperature coefficient,

$f_{1}=f_{2}=1.00$ - nonsynchronous rotation coefficients of the components,

$\beta_{1}=\beta_{2}=0.08$ - gravity-darkening coefficients of the components,

$u_{1}=0.66$ - limb-darkening coefficient of the primary.

Note: No. - data set No., $\theta_{1,2}$ - spotted areas angular dimensions, $\lambda_{1,2}$ - spots longitude and $\varphi_{1,2}$ - spots latitude (all in degrees); $F_{1}, F_{2}$ filling coeficients for critical Roche lobes of the primary and secondary, $T_{2}$ - temperature of the secondary, $i$ - orbit inclination (in degrees), $u_{2}$ - limb-darkening coefficient of the secondary, $\Omega_{1}, \Omega_{2}$ - dimensionless surface potentials of the primary and secondary, $R_{1}$, $R_{2}$ - stellar polar radii in units of the distance between the component centres and $\Sigma(\mathrm{O}-\mathrm{C})^{2}-$ final sum of squares of residuals between observed (LCO) and synthetic (LCC) light curves.

differential-rotation effect. As seen from the Fig. 2e, and Fig. 3, the light curve depression is shifted through the orbital phase with time. This depression migration can be explained by the difference between the spotted area angular velocity $\omega$ and the Keplerian angular velocity in the system's revolution $\omega_{\mathrm{K}}$. This is to be expected in the case of the differential rotation of the stellar surface layers and for the nonsynchronous rotation of the components. Here a short-period CB is analysed whose stars fill in their critical Roche lobes significantly; hence due to the tidal effects the rotation of the components is expected to be synchronous.

The depression shifting along the light curves can be in both senses, direct and retrograde (see Busso et al. 1984), which depends on whether the spotted area latitude exceeds the corotational one or not. This circumstance (shifting sense) appears as a serious indication in favour of the diferential rotation existence. By applying simple approximations, based on the analogy with the problem of the differential rotation of the Sun, it is possible in certain cases to obtain the data on the component differential rotation in $R S C V n$ type of CBs. Following the idea applied by Busso et al. (1985) the active region is approximated here by a circular spotted area whose centre corresponds to the photocentre of the given region and the photometric time variations of the light curves are interpreted through the change in the position and size of the spot. Due to the meridional motion from higher towards lower latitudes, the differential rotation causes the shift of the spotted area longitude in the course of time. The differential-rotation parameters are derived on the basis of these longitude shifts.

The single spot model is compatible with that for obtaining the differential-rotation parameters. Although it has been shown that this model has certain drawbacks, in analysing the differential rotation one can exploit the data of the spotted area longitudes (see Table 2) for the entire set of observed light curves over the interval 1973-1981. Such an analysis is presented in Djurašević (1996). The nonlinear system of equations, describing the differential rotation is solved following adequate approaches (Marquardt's algorithm - Marquardt 1963 - and SIMPLEX - Torczon 1991). These algorithms are suitable for the problems of nonlinear optimisation and they enable one to solve equation systems describing the differential rotation. The results obtained for the case of $S \mathrm{~V} \mathrm{Cam}$ (Djurašević 1996) indicate that the spotted area appears at high latitudes (about $84^{\circ}$ ), whereas the corotation latitude is about $30^{\circ}$. Although the model for calculating the differential-rotation parameters is rough, this result is an indication that in the case of the short-period $R S C V n$ type of $\mathrm{CBs}$, spotted areas can be expected in the polar regions. 


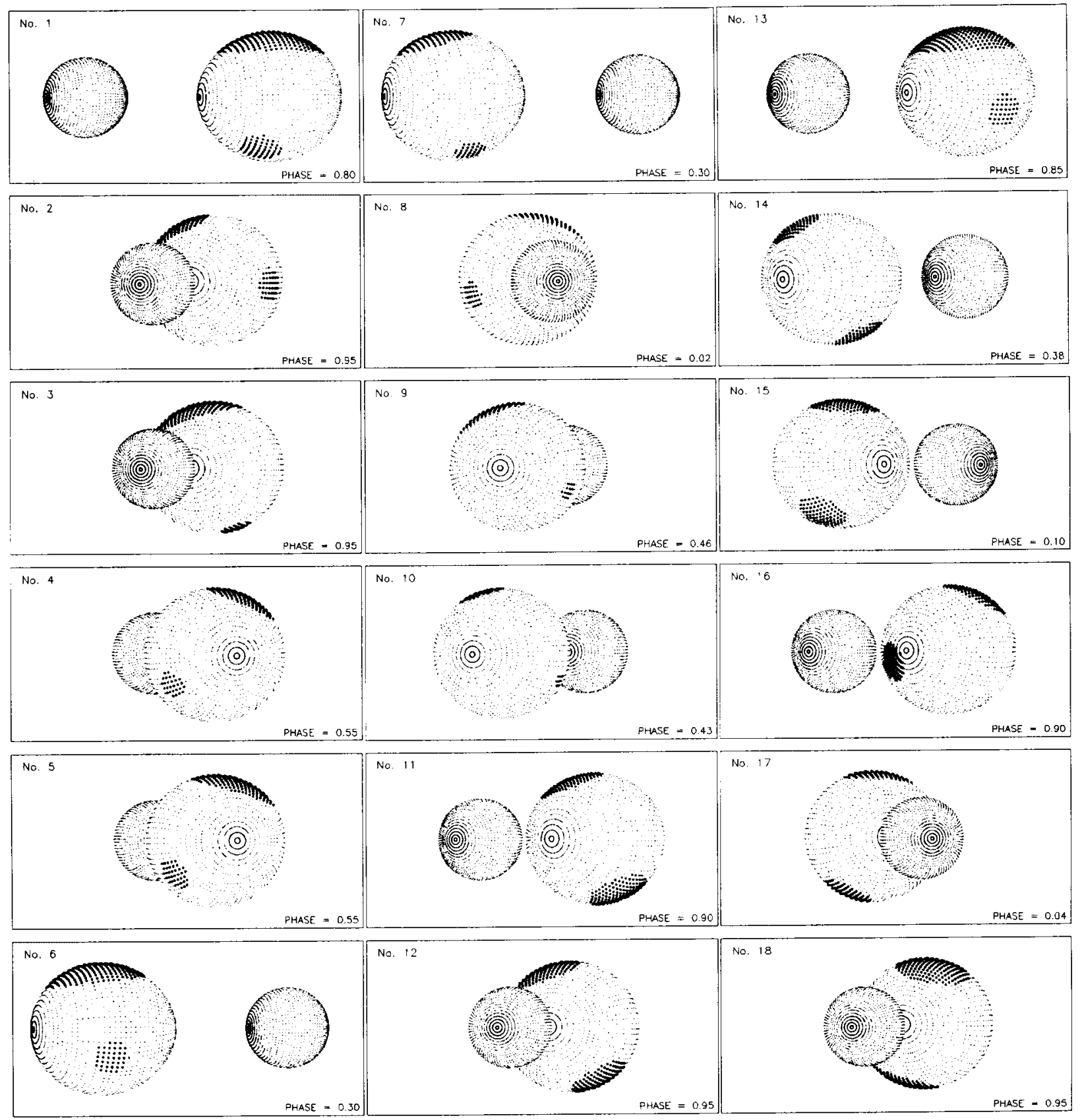

Fig. 4. The view of the CB $S V$ Cam at corresponding orbital phase with parameters obtained by solving the inverse problem

The consideration of the differential rotation problem and the results of the light curve analysis for $S V$ Cam presented here, clearly indicate that large spotted areas in the polar regions of the primary could be accepted as a reality.

Acknowledgements. This work has been supported by the Ministry for Sciences and Technology of Serbia through the project "Astrometrical, Astrodynamical and Astrophysical investigations".

\section{References}

Budding E., 1977, Ap\&SS 48, 207

Budding E., Zeilik M., 1987, ApJ 319, 827

Budding E., 1973, Ap\&SS 22, 87

Budding E., Najim N.N., 1980, Ap\&SS 72, 369

Busso M., Scaltriti F., Blanco C., et al., 1984, A\&A 135, 255

Busso M., Scaltriti F., Cellino A., 1985, A\&A 148, 29

Cellino A., Scaltriti F., Busso M., 1985, A\&A 144, 315

Díaz-Cordovés J., Claret A., Giménez A., 1995, A\&AS 110, 329

Djurašević G., 1991, Publ. Obs. Astron. Belgrade 42, 1 
Djurašević G., 1992a, Ap\&SS 196, 241

Djurašević G., 1992b, Ap\&SS 197, 17

Djurašević G., 1996, Ap\&SS (accepted)

Eaton J.A., Hall D.S., 1979, ApJ 227, 907

Hall D.S., 1976, in IAU Coll. 29, Multiple Periodic Variable Stars, Fitch W.S. (ed.). Reidel, Dordrecht, p. 287

Hilditch R.W., Harland D.M., McLean B.J., 1979, MNRAS 187,797

Lucy L.B., 1967, Zs. f. Ap. 65, 89
Marquardt D.W., 1963, J. Soc. Ind. Appl. Math. 11, 431

Osaki Y., 1970, MNRAS 148, 391

Patkós L., 1981, Ap. Lett. 22, 1

Patkós L., 1982, Comm. Konkoly Obs., No. 80

Schüssler M., Solanski S.K., 1992, A\&A 264, L13

Torczon V., 1991, SIAM J. Optimization 1, 123

Wilson R.E., Devinney E.J., 1971, ApJ 166, 605

Zeilik M., De Blasi C., Rhodes M., Budding E., 1988, ApJ 332, 293 